\title{
Effect of tumor necrosis factor inhibitors on interstitial lung disease in rheumatoid arthritis: angel or demon?
}

This article was published in the following Dove Press journal:

Drug Design, Development and Therapy

\section{Ying Huang \\ Weiji Lin \\ Zhe Chen \\ Yu Wang \\ Yao Huang \\ Shenghao Tu}

Institute of Integrated Traditional Chinese and Western Medicine, Tongji Hospital, Tongji Medical College, Huazhong University of Science and Technology, Wuhan, People's Republic of China
Correspondence: Shenghao Tu Institute of Integrated Traditional Chinese and Western Medicine, Tongji Hospital, Tongji Medical College, Huazhong University of Science and Technology, Jiefang Avenue 1095, Wuhan 430022,

People's Republic of China

Tel +862783663402

Email shtu@tjh.tjmu.edu.cn
Objectives: This study evaluated the correlation between tumor necrosis factor alpha inhibitor (TNF-I) and interstitial lung disease (ILD) in rheumatoid arthritis (RA). We aimed to raise awareness and consummate therapy by summarizing the characteristics of the adverse events of ILD.

Methods: A comprehensive search of the PubMed, Embase, Ovid, Cochrane, China National Knowledge Infrastructure, and Wanfang databases was performed from inception to November 2018. Statistical analysis of demographic characteristics, clinical features, and relative risks was performed using Microsoft Excel 2007 and SPSS version 20.0.

Results: A total of 7 eligible articles and another 28 case reports were enrolled. The 7 cohort studies demonstrated the tendency that ILD cases might not benefit from TNF-I therapy. TNF-I might be associated with ILD adverse events. The case reports further confirmed these findings, as most $(87.5 \%)$ of the cases showed that TNF-1 was harmful to patients with ILD and even resulted in a 35\% mortality rate. Further investigation revealed that ILD adverse events tended to appear in female patients with a long RA history $(p<0.05)$. The subgroup analysis suggested that early detection and precise treatment are key factors in determining survival or death when an ILD adverse event occurs. A large proportion of ILD adverse events $(48.6 \%)$ appeared at $2.38 \pm 1.03$ weeks after the infusion of infliximab.

Conclusion: A fresh look at the evidence highlights that TNF-I might be associated with ILD adverse events in RA, which can induce more severe pulmonary symptoms and even result in death. Therefore, more attention should be paid to effective prevention, early diagnosis, and precise management. Notably, further prospective cohort studies are warranted to better interpret the association or causality between TNF-I and ILD.

Keywords: interstitial lung disease, rheumatoid arthritis, tumor necrosis factor inhibitors, adverse effects

\section{Introduction}

Interstitial lung disease (ILD) is characterized by diffuse pulmonary parenchyma, alveolar inflammation, and interstitial fibrosis. The incidence of ILD was $20-30 \%$ in patients with rheumatoid arthritis (RA) according to the study by Castelino et al. ${ }^{1}$ ILD commonly occurs as an extra-articular manifestation of severe $\mathrm{RA}^{2}{ }^{2}$ and results in high morbidity and mortality. ${ }^{3,4}$ Additionally, the prevalence of ILD has been confirmed to increase by $0.3-11 \%$ in RA treated with methotrexate (MTX), ${ }^{5,6}$ which serves as a reminder that more caution should be taken when using other disease-modifying drugs (DMARDs). Although TNF-I has been widely used in the 
past decades, its adverse effects on non-infectious complications (especially ILD) have not been well recognized and warrant investigation.

Initially, we presumed that ILD in patients with RA may benefit from TNF-I because TNF- $\alpha$ plays a pivotal role in the pathogenesis of ILD. $^{7} \mathrm{~A}$ case report from the same period also indicated that TNF-1 has a positive effect on ILD. ${ }^{8}$ The results of clinical randomized controlled trials further emphasized that there is no difference compared with other DMARDs in terms of ILD. However, an ever-increasing incidence of adverse events has been reported since the first report of ILD induction by infliximab in 2002 buchong cankao wenxian. The randomized controlled trials performed thus far do not provide enough guidance. Therefore, we performed a systematic literature research and attempted to summarize the commonalities in order to better characterize the magnitude of this problem.

In this review, we discuss the risks of TNF-I use with respect to new onset of RA-ILD, the deterioration of RAILD, and the mortality of RA-ILD based on clinical cohort studies and case reports. We attempted to summarize the clinical characteristics of ILD adverse events, and to distinguish ILD secondary to TNF-I therapy from spontaneous ILD. Lastly, we hope to raise more awareness, enhance the diagnosis, improve the therapy, and fill the gaps in current knowledge.

\section{Methods}

\section{Search strategy}

The PubMed, Embase, Ovid, Cochrane, China National Knowledge Infrastructure, and Wanfang databases were searched using the keywords "etanercept", "infliximab", "adalimumab", "golimumab", "certolizumab pegol", "tumour necrosis factor inhibitors", "anti-TNF", "biologic disease-modifying anti-rheumatic drugs", "lung diseases, interstitial", and "pulmonary fibrosis", for articles published up to November 2018 according to PRISMA guidelines. The relevant references of the selected articles were also hand searched to retrieve additional eligible articles.

\section{Inclusion and exclusion criteria}

The studies included in this systematic review had to meet all of the following inclusion criteria: (1) case-control studies, cohort studies, clinical trials, and case reports; (2) Chest CT as used to diagnose ILD, RA was diagnosed according to American College of Rheumatology European Union Against Rheumatism classification criteria for RA (2010)
(3) presenting enough information about RA-ILD, TNF-I, and the relationship between them; (4) investigating the risk factors of RA-ILD (age, sex, smoking, antibody seropositivity, and others); and (5)written in the English language. The exclusion criteria were as follows: (1) basic studies, mechanistic studies, review articles, guidelines, and meta-analysis; (2) duplicate data presented in multiple studies, unavailability of full-text articles, and lack of necessary data; (3) included patients less than18 years old or pregnant women, and (4) Except for the simple RA.

\section{Data extraction and statistical analysis}

Two authors (Huang and Lin) independently determined the eligible studies according to the aforementioned criteria, extracted the required information, and addressed any discrepancies through a discussion.

\section{Statistical analysis}

Statistical analysis was carried out using Microsoft Excel 2007 (Microsoft Corporation, Redmond, WA, USA) and IBM SPSS Statistics 20.0 (IBM Corporation, Armonk, NY, USA), Chi-square test was used for statistical analysis.

\section{Results}

\section{Literature search}

As shown in Figure 1, a total of 1506 articles were identified through the database searching. We scored 1304 studies after the removal of duplicates; 1182 articles not relevant to our topic were excluded out of the systematic review after reviewing the titles. The remaining 122 articles underwent detailed reading of abstracts, among which 78 articles were further excluded according to the inclusion and exclusion criteria. Because 9 of the remaining 44 articles were not available of full-text, it's a pity finally we only get 7 eligible articles $(22,981$ patients) and another 28 case reports (40 patients). All of the selected studies were published between 2002 and 2018. More details about the eligible articles are provided in Table 1, and details about the case reports are shown in Table 2. We performed a descriptive review rather than a meta-analysis owing to the significant heterogeneity and the limited data.

\section{Controversy and consensus in}

\section{large-sample studies}

As shown in Table 1 , Herrinton et $\mathrm{al}^{9}$ provided evidence that TNF-I does not increase the incidence of ILD in 


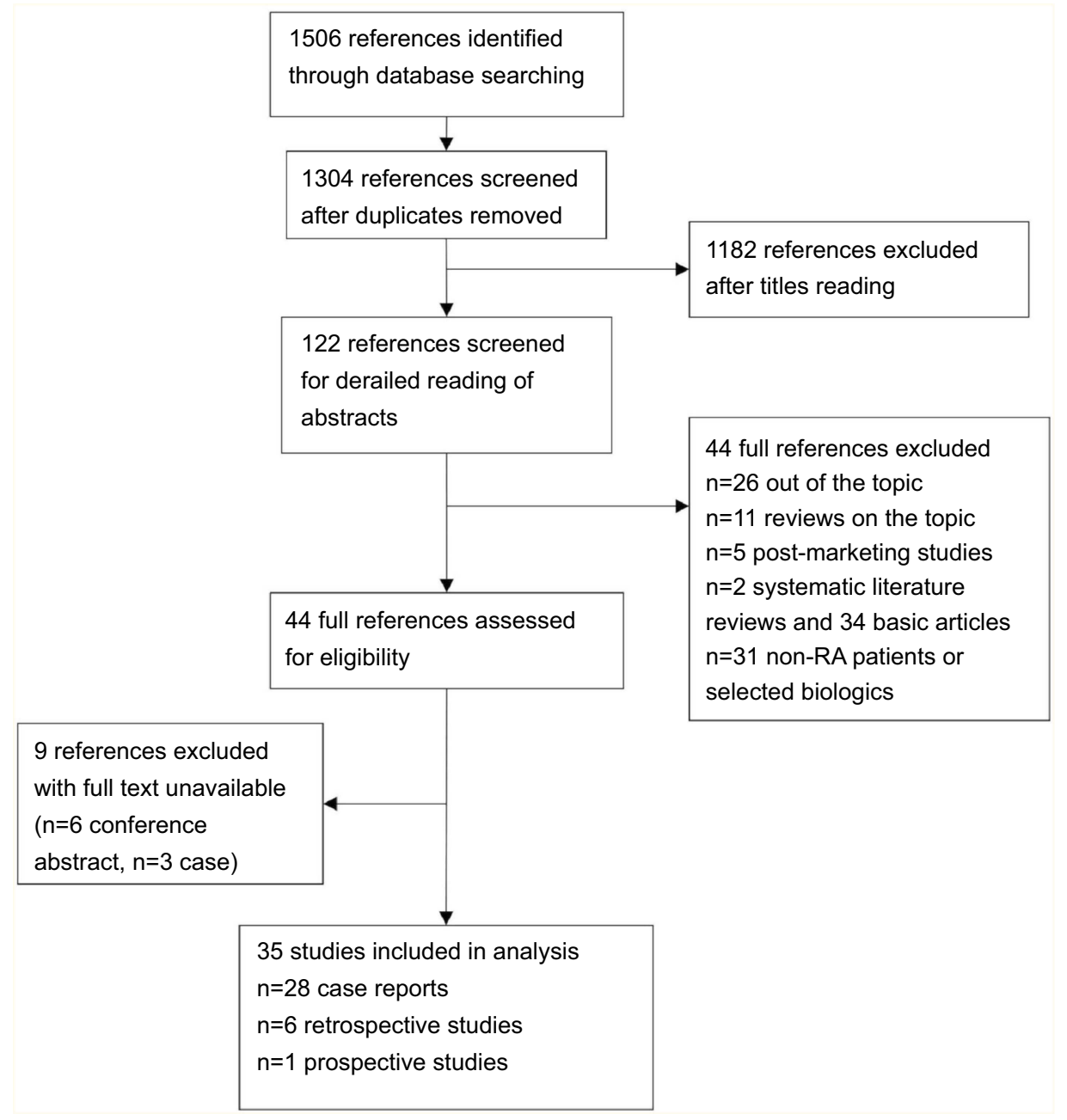

Figure I Search parameters and strategies of literature review.

autoimmune diseases, mainly in RA. However, Nakashita et $\mathrm{al}^{10}$ suggested that TNF-I has the potential risk of inducing ILD and might exacerbate pre-existing ILD compared with non-TNF-I biological therapy. Moreover, their further study ${ }^{11}$ emphasized that TNF-I was less effective than abatacept in patients with pre-existing ILD. In the same year, Curtis et $\mathrm{al}^{12}$ reported that no significant risks of ILD and related complications were observed with TNF-I when compared with T-cell, B-cell, and interleukin (IL)-6 inhibitors. In a small-sample study, Koo et $\mathrm{al}^{13}$ emphasized that a potential risk of mortality existed in older patients with RA-ILD within months of the initial TNF-I therapy. In 2017, Druce et $\mathrm{al}^{14}$ hypothesized that patients with RA-ILD using TNF-I had a higher mortality rate than patients using rituximab. Additionally, Detorakis et $\mathrm{al}^{15}$ compared TNF-I with MTX and then provided supporting evidence for the efficacy and safety of TNF-I according to high-resolution computed tomography (HRCT) evaluation and pulmonary function tests ${ }^{15}$ and compared TNF-I with MTX. In short, previous studies demonstrated the tendency that ILD might not benefit from TNF-I therapy.

\section{Noteworthy differences in case reports}

As depicted in Table 3, we found that ILD adverse events after TNF-I therapy accounted for $87.5 \%$ of the retrieved cases. A $68.57 \%$ rate of positivity to rheumatoid factor or anti-cyclic citrullinated peptide antibody was mentioned in the known case reports, and most patients presented an invasive disease. Initially, we speculated that age, sex, RA history, pulmonary history, use of MTX, and the different types of TNF-I led to different outcomes. After the statistical analysis (chi-square test) of retrieved cases, we conjectured that TNF-I is beneficial or harmful to ILD depending on the history of RA and $\operatorname{sex}(p<0.05)$, rather than age, use of MTX, and other factors. A female preponderance $(74.29 \%)$ was observed in the spontaneous ILD adverse events, whereas patients treated with TNF-I 


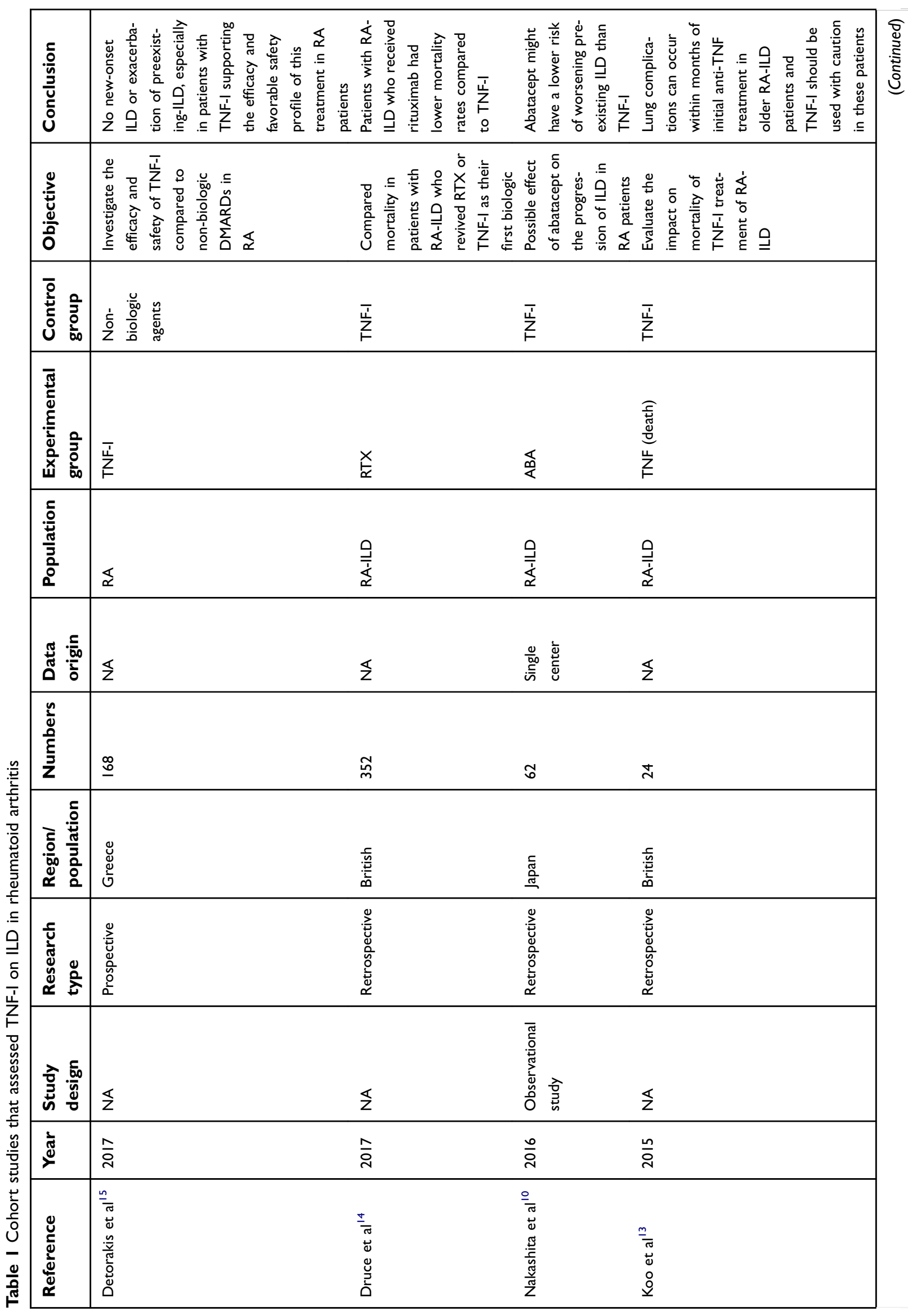




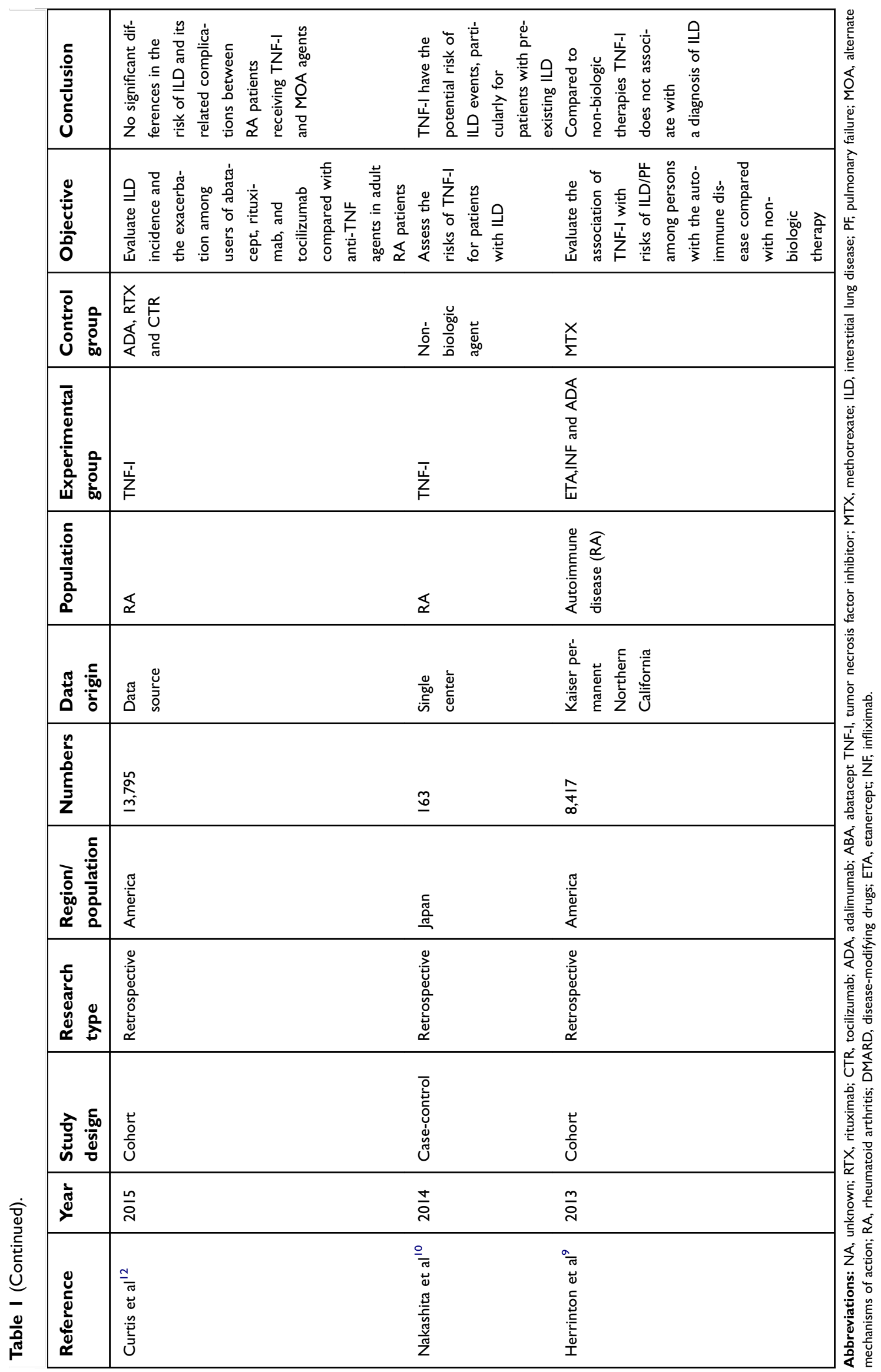




\begin{tabular}{|c|c|c|c|c|c|c|c|c|c|c|c|c|c|c|}
\hline$\sim$ & 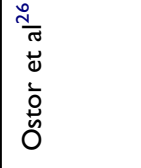 & ષ્ণ & $\hat{o}$ & $\Sigma$ & $\Sigma$ & 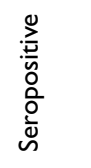 & + & 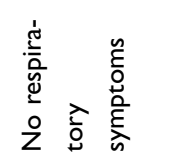 & 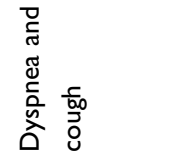 & $\begin{array}{l}\frac{\grave{y}}{4} \\
\frac{0}{5} \\
\frac{0}{3} \\
m \\
m \\
m\end{array}$ & 은 & $\underline{\underline{\Sigma}}$ & 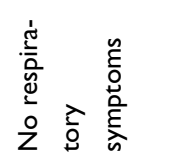 & 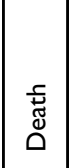 \\
\hline$n$ & 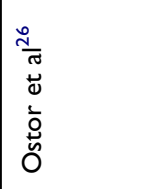 & ঃั & $\bar{\sigma}$ & ч & $\overleftarrow{z}$ & 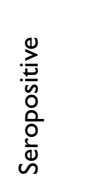 & $\simeq$ & 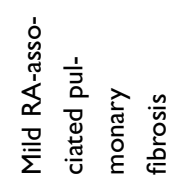 & 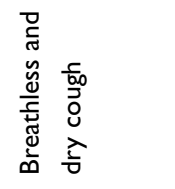 & 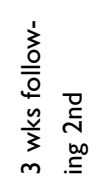 & $\stackrel{0}{z}$ & $\underline{\underline{u}}$ & 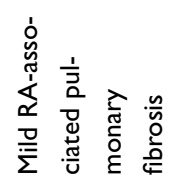 & 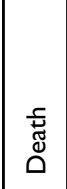 \\
\hline N & 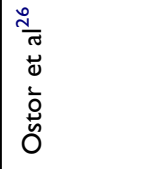 & ठั & $q$ & ч & ó & 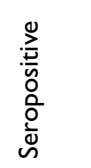 & $\approx$ & 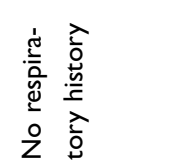 & 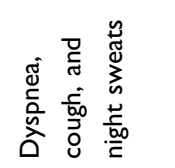 & 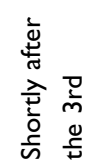 & $\stackrel{\circ}{z}$ & $\underline{\underline{\underline{z}}}$ & 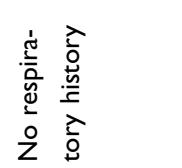 & 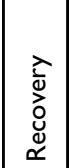 \\
\hline 0 & 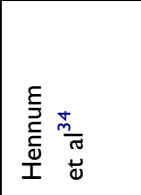 & ¿্ণ & ถั & $\Sigma$ & 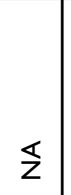 & 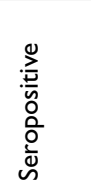 & 0 & 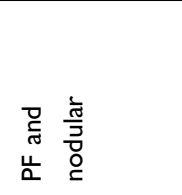 & 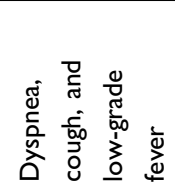 & 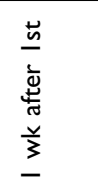 & $\stackrel{\check{\Xi}}{\nu}$ & $\underline{\underline{u}}$ & 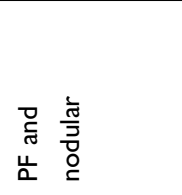 & 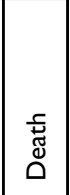 \\
\hline in & 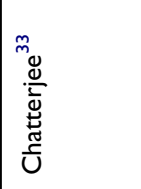 & ठั & 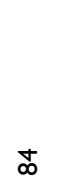 & 4 & 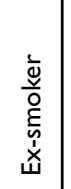 & 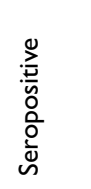 & 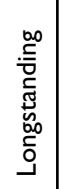 & 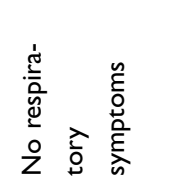 & 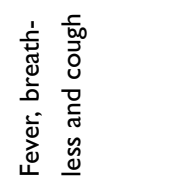 & 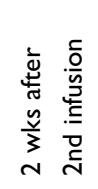 & zo & $\underline{\underline{u}}$ & 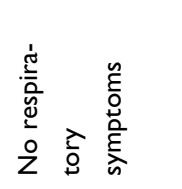 & 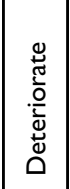 \\
\hline$\nabla$ & 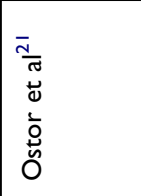 & 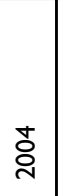 & $\stackrel{n}{\kappa}$ & ч & $\S$ & $\mathbb{Z}$ & $m$ & 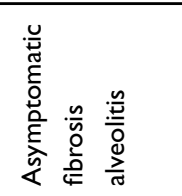 & 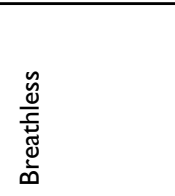 & 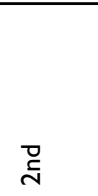 & $\stackrel{\circ}{z}$ & $\underline{\underline{u}}$ & 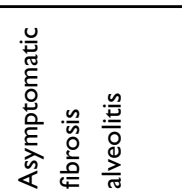 & 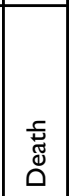 \\
\hline$\nabla$ & 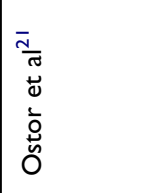 & 芯 & $\hat{0}$ & $\Sigma$ & $\mathbb{z}$ & $\S$ & $\nabla$ & 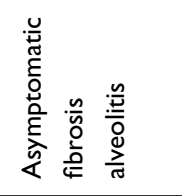 & 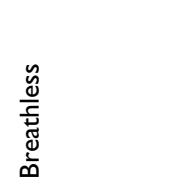 & $\stackrel{p}{m}$ & zo & $\underline{\underline{u}}$ & 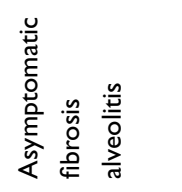 & 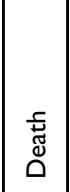 \\
\hline$\nabla$ & 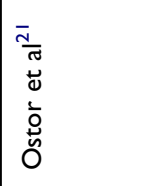 & 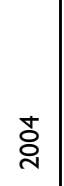 & 8 & ч & 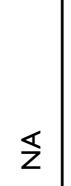 & $\mathbb{z}$ & $\simeq$ & 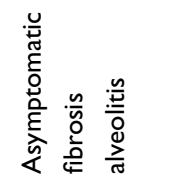 & 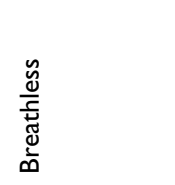 & 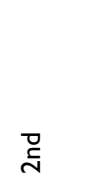 & $\stackrel{\circ}{z}$ & $\underline{\underline{u}}$ & 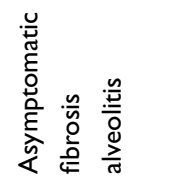 & 点 \\
\hline$m$ & 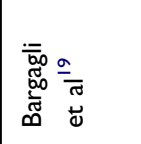 & 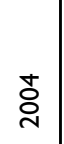 & R & ч & ò & $\mathbb{z}$ & $\mathbb{z}$ & 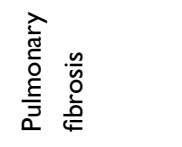 & $\S$ & 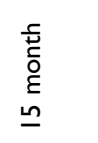 & $\stackrel{\circ}{z}$ & $\underline{\underline{u}}$ & 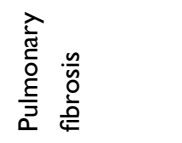 & 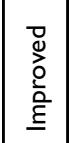 \\
\hline$N$ & 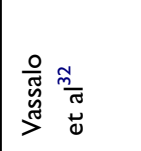 & ర్లి & $\pi$ & $\Sigma$ & 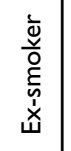 & 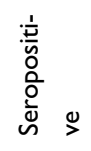 & $m$ & 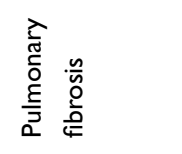 & 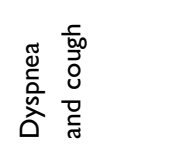 & $\stackrel{\bar{\Xi}}{\stackrel{\bar{\Xi}}{\beth}}$ & $\stackrel{\check{y}}{\nu}$ & $\underline{\underline{u}}$ & 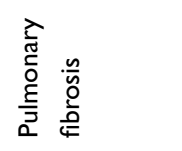 & 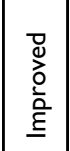 \\
\hline- & 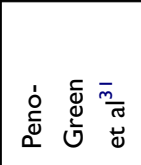 & ర్రి & 오 & ч & $\S$ & 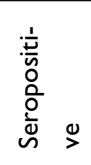 & in & $\S$ & 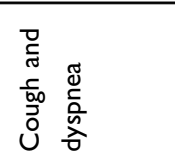 & $\underset{\stackrel{c}{o}}{\stackrel{\sim}{~}}$ & $\stackrel{\check{\Xi}}{\check{\nu}}$ & 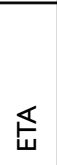 & 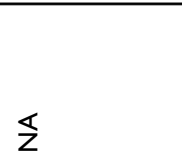 & 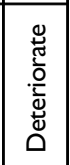 \\
\hline & 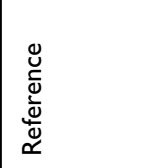 & 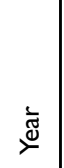 & $\underset{8}{\stackrel{8}{<}}$ & હ & 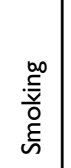 & $\begin{array}{l}\nwarrow \\
\grave{o} \\
\grave{U}\end{array}$ & 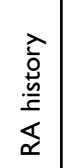 & 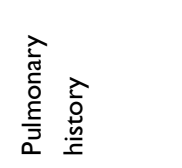 & 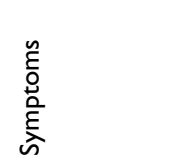 & 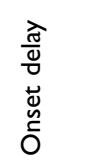 & $\stackrel{\times}{\stackrel{x}{\Sigma}}$ & 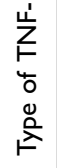 & 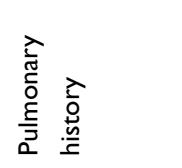 & 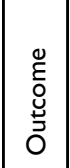 \\
\hline
\end{tabular}




\begin{tabular}{|c|c|c|c|c|c|c|c|c|c|c|c|c|c|c|}
\hline$\underline{m}$ & 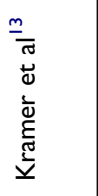 & ర్రి & 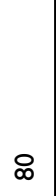 & ч & $\S$ & 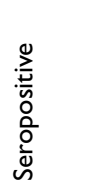 & $\Sigma$ & $\S$ & 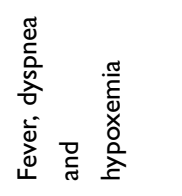 & 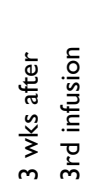 & $\stackrel{\check{\Xi}}{\tau}$ & $\underline{\underline{u}}$ & $\S$ & 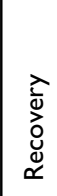 \\
\hline$\underline{m}$ & 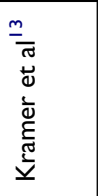 & ర్రి & t & ч & $\mathbb{z}$ & 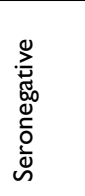 & 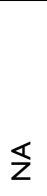 & $\begin{array}{l}\frac{1}{\frac{\pi}{3}} \\
\frac{\bar{y}}{2} \\
\frac{2}{z}\end{array}$ & 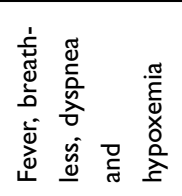 & 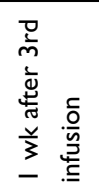 & $\stackrel{\tilde{u}}{\check{\nu}}$ & $\underline{\underline{u}}$ & $\begin{array}{l}\frac{1}{\frac{1}{n}} \\
\frac{\bar{z}}{0} \\
\frac{0}{2}\end{array}$ & 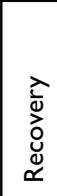 \\
\hline$\underline{m}$ & 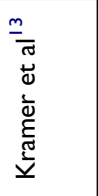 & ర్రి & 3 & 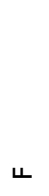 & $\mathbb{z}$ & 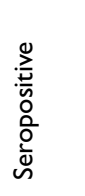 & 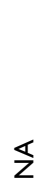 & $\S$ & 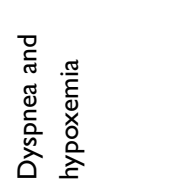 & 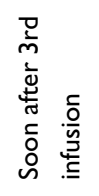 & 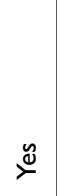 & $\underline{\underline{u}}$ & $\mathbb{z}$ & 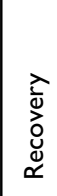 \\
\hline$\simeq$ & 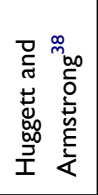 & ঃั & $\stackrel{\circ}{\circ}$ & ч & ò & 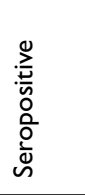 & $m$ & 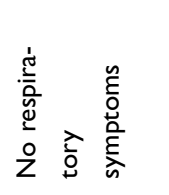 & 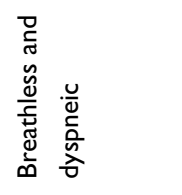 & $\begin{array}{l}\frac{y}{3} \\
0\end{array}$ & $\stackrel{\tilde{\Perp}}{\nu}$ & 造 & 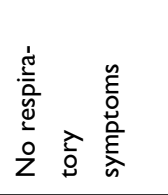 & 䓌 \\
\hline$=$ & 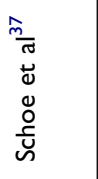 & ષัণ & $\hat{o}$ & $\Sigma$ & 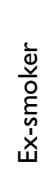 & 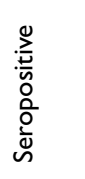 & $\sigma$ & $\S$ & 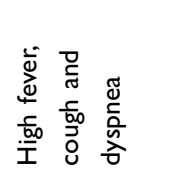 & $\begin{array}{l}\frac{y}{3} \\
\simeq\end{array}$ & $\stackrel{\check{\Xi}}{\check{\nu}}$ & 这 & $\S$ & 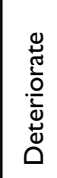 \\
\hline 으 & 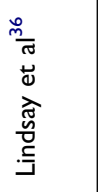 & రัరి & t & ч & 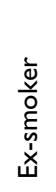 & 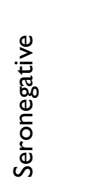 & $=$ & 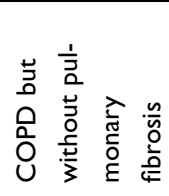 & 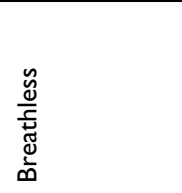 & 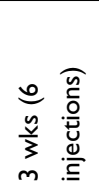 & 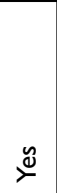 & $\underset{w}{\overleftarrow{E}}$ & 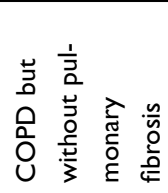 & 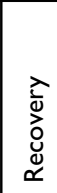 \\
\hline 으 & 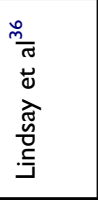 & ઠัర & $\bar{\sigma}$ & \llcorner & 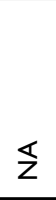 & ¿ & 으 & 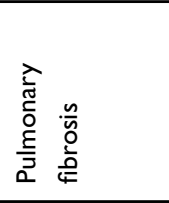 & 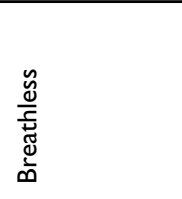 & 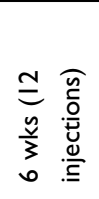 & $\stackrel{\check{\Perp}}{\nu}$ & 芷 & 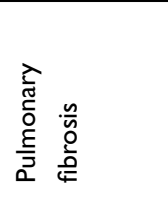 & 䓌 \\
\hline$a$ & 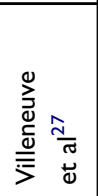 & ठ্ণ & $R$ & $\Sigma$ & 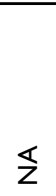 & 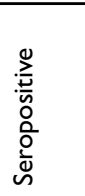 & $\simeq$ & 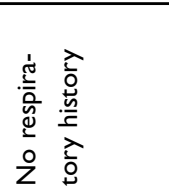 & 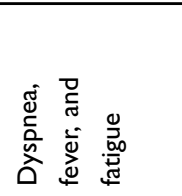 & 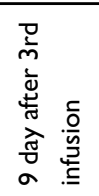 & 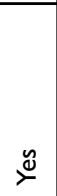 & $\underline{\underline{u}}$ & 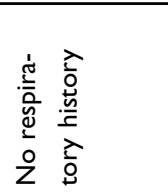 & 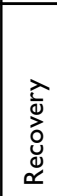 \\
\hline$\infty$ & 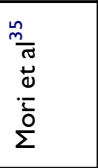 & ঃั & $\stackrel{\circ}{\circ}$ & 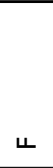 & 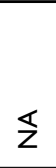 & 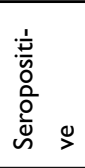 & n & 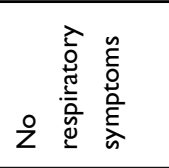 & 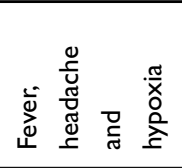 & 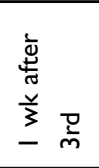 & $\stackrel{\tilde{v}}{\nu}$ & $\underline{\underline{u}}$ & 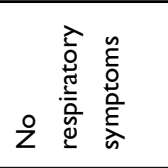 & 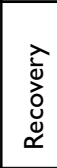 \\
\hline n & 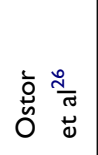 & ఫัరి & $\stackrel{n}{\wedge}$ & ч & $\begin{array}{l}\overline{\bar{u}} \\
\overline{0} \\
\overline{\bar{\nu}} \\
\dot{凶}\end{array}$ & 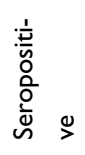 & m & 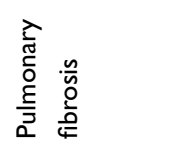 & 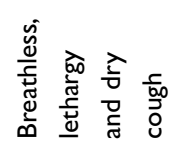 & \begin{tabular}{ll} 
& \multicolumn{0}{c}{} \\
$\frac{1}{0}$ & \\
$\frac{0}{0}$ & 0 \\
$\frac{0}{3}$ & $\frac{5}{3}$ \\
- & $\underline{0}$
\end{tabular} & $\stackrel{0}{z}$ & $\underline{\underline{u}}$ & 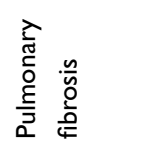 & 䓌 \\
\hline n & 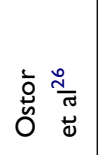 & ઠัర & $\stackrel{\infty}{\infty}$ & ч & 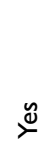 & 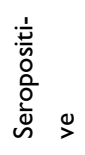 & - & 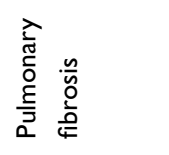 & 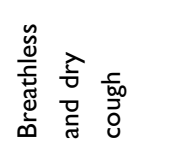 & 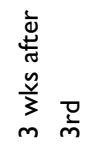 & $\stackrel{0}{z}$ & $\underline{\underline{u}}$ & 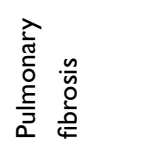 & 䍃 \\
\hline & 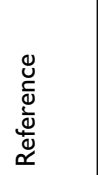 & 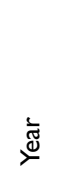 & $\underset{\mathbb{<}}{\mathbb{8}}$ & ঐ & 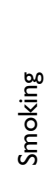 & $\begin{array}{l}\overleftarrow{x} \\
\dot{0} \\
\grave{U}\end{array}$ & 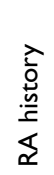 & 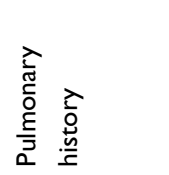 & 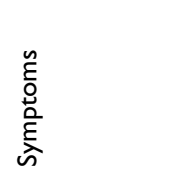 & 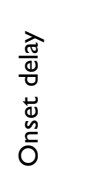 & $\stackrel{\times}{\stackrel{\bullet}{E}}$ & 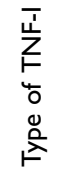 & 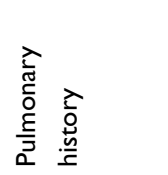 & 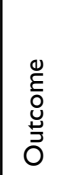 \\
\hline
\end{tabular}




\begin{tabular}{|c|c|c|c|c|c|c|c|c|c|c|c|c|c|c|}
\hline $\bar{N}$ & 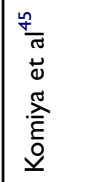 & $\overline{\bar{N}}$ & $\stackrel{\infty}{\wedge}$ & $\Sigma$ & $\S$ & 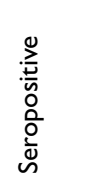 & $\underline{\infty}$ & 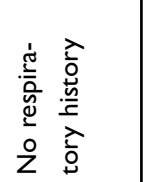 & 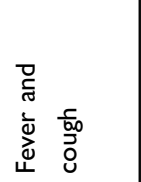 & 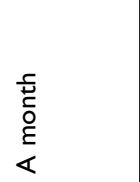 & $\stackrel{\mathscr{\nu}}{\check{\nu}}$ & 希 & 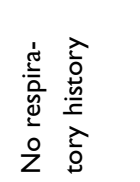 & 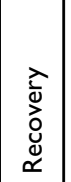 \\
\hline 유 & 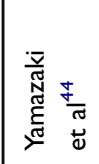 & 음 & $\stackrel{t}{0}$ & ч & $\S$ & 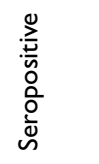 & $\underline{\infty}$ & 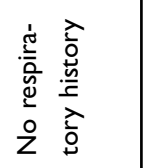 & 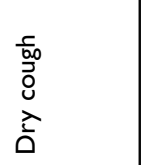 & $\begin{array}{l}\frac{n}{3} \\
\stackrel{N}{N}\end{array}$ & $\stackrel{\mathscr{y}}{\check{\nu}}$ & 造 & 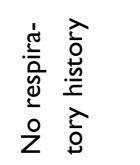 & 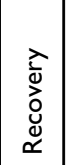 \\
\hline$\underline{a}$ & 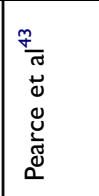 & $\stackrel{\sim}{\circ}$ & $\pi$ & ч & $\stackrel{\check{0}}{z}$ & 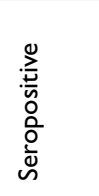 & $\infty$ & 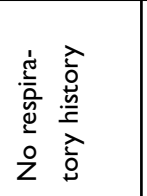 & 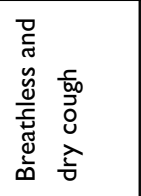 & $\begin{array}{l}\frac{n}{3} \\
\frac{\sigma}{\sigma}\end{array}$ & 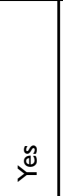 & U্ষ & 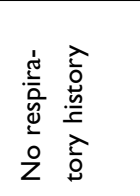 & 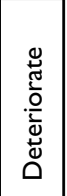 \\
\hline$\underline{\infty}$ & 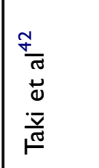 & ઠి & $\stackrel{\nabla}{N}$ & ч & $\S$ & 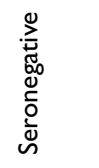 & $\mathbb{z}$ & 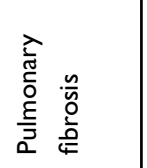 & 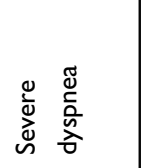 & 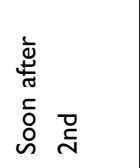 & 은 & $\underline{\underline{u}}$ & 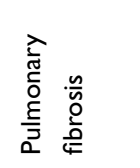 & 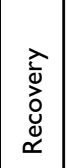 \\
\hline$\Sigma$ & 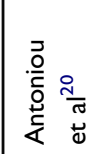 & ఫે & $R$ & $\Sigma$ & 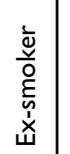 & 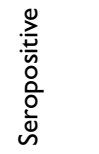 & in & 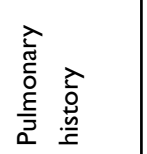 & 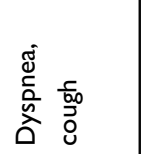 & $\underline{\lambda}$ & $\stackrel{\mathscr{y}}{\longleftarrow}$ & $\underline{\underline{\underline{u}}}$ & 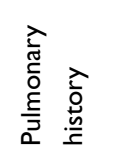 & 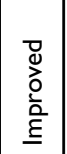 \\
\hline$\simeq$ & 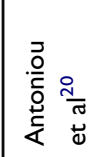 & 官 & 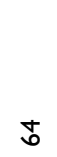 & $\Sigma$ & $\stackrel{\check{\nu}}{\check{\nu}}$ & 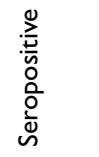 & $N$ & 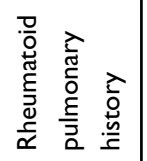 & 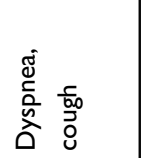 & $\underline{\lambda}$ & $\stackrel{\mathscr{\Xi}}{\check{\nu}}$ & $\underline{\underline{u}}$ & 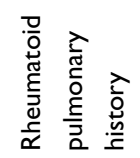 & 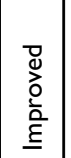 \\
\hline$\simeq$ & 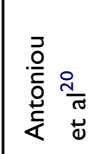 & 官 & ธิ & $\Sigma$ & 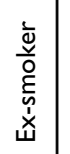 & 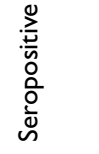 & 으 & 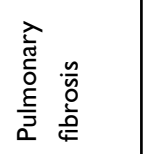 & 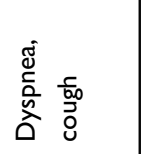 & $\underline{-}$ & 은 & $\underline{\underline{\underline{u}}}$ & 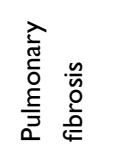 & 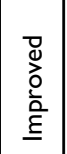 \\
\hline$\simeq$ & 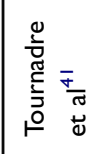 & 仓े & กี & $\Sigma$ & $\stackrel{\check{\varpi}}{\check{\nu}}$ & 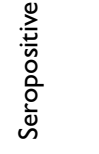 & in & 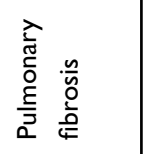 & 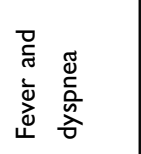 & $\begin{array}{l}\frac{y}{3} \\
\vec{v}\end{array}$ & $\stackrel{\varpi}{\check{\nu}}$ & 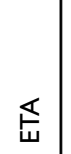 & 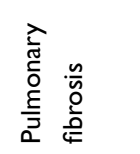 & 带 \\
\hline$\stackrel{0}{0}$ & 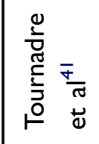 & 官 & $\mathcal{F}$ & $\Sigma$ & $\stackrel{\check{o}}{z}$ & 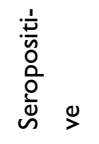 & $\simeq$ & 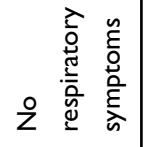 & 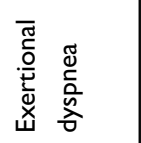 & $\begin{array}{l}\frac{n}{3} \\
0 \\
0\end{array}$ & $\stackrel{\check{\varpi}}{\check{\nu}}$ & 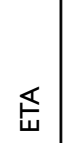 & 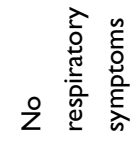 & 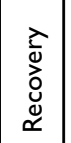 \\
\hline$\underline{\underline{n}}$ & 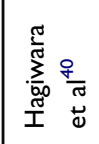 & ఫิ & R & ч & 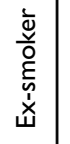 & 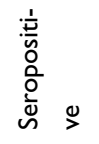 & 0 & 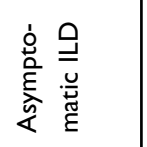 & 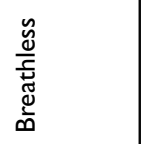 & $\begin{array}{l}\frac{y}{3} \\
\infty\end{array}$ & $\stackrel{\check{y}}{\check{\nu}}$ & 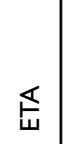 & 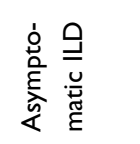 & 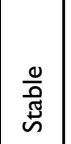 \\
\hline \pm & 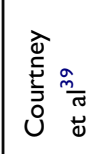 & ¿ัరి & 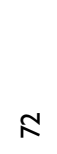 & ч & $\S$ & $\mathbb{z}$ & 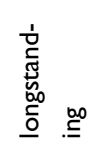 & 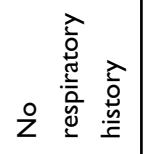 & 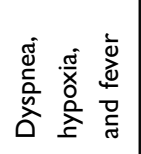 & 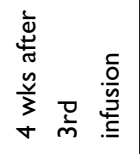 & 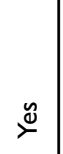 & $\underline{\underline{u}}$ & 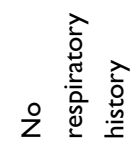 & $\begin{array}{l}\text { 哥 } \\
\text { 口. }\end{array}$ \\
\hline & 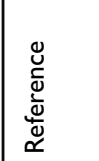 & 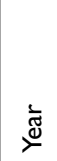 & $\underset{\leftarrow}{\stackrel{\Xi}{\alpha}}$ & $\stackrel{x}{凶}$ & 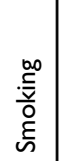 & 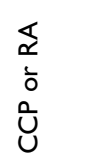 & 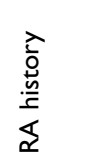 & 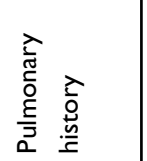 & 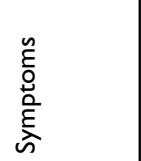 & 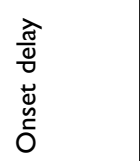 & $\underset{上}{\stackrel{\bullet}{E}}$ & 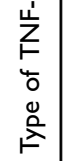 & 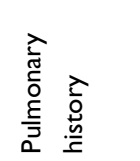 & 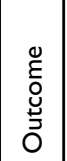 \\
\hline
\end{tabular}




\begin{tabular}{|c|c|c|c|c|c|c|c|c|c|c|c|c|c|c|}
\hline$\stackrel{\infty}{\sim}$ & 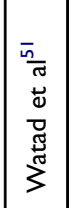 & 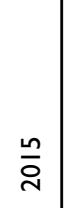 & ๓ & ч & $\begin{array}{l}\delta \\
z \\
z\end{array}$ & $\Sigma$ & $\stackrel{\circ}{ }$ & 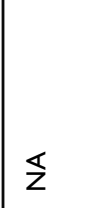 & 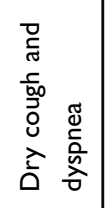 & $\begin{array}{l}\underline{\underline{y}} \\
\underline{3} \\
\underline{0}\end{array}$ & 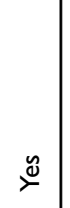 & $\underset{\Psi}{\mathbb{E}}$ & $\mathbb{Z}$ & 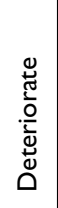 \\
\hline$\tilde{N}$ & 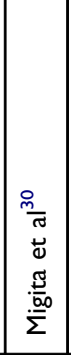 & $\overline{\bar{N}}$ & 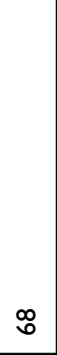 & 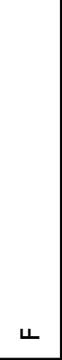 & $\underbrace{\mathscr{y}}$ & $\mathbb{z}$ & $\underline{n}$ & 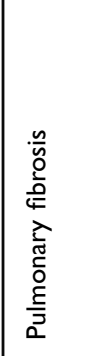 & 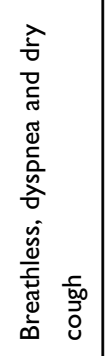 & $\begin{array}{l}\frac{n}{3} \\
m\end{array}$ & $\stackrel{\circ}{z}$ & ü & 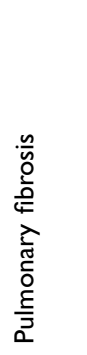 & 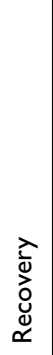 \\
\hline$\stackrel{2}{*}$ & 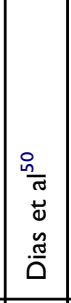 & $\stackrel{m}{i}$ & ธ & 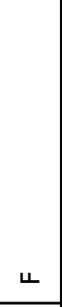 & $\S$ & $\mathbb{z}$ & i & 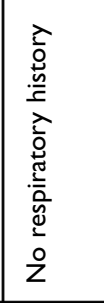 & 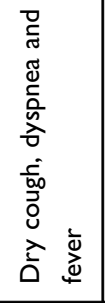 & 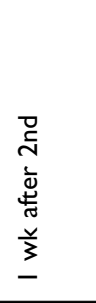 & $\stackrel{\mathscr{x}}{x}$ & 市 & 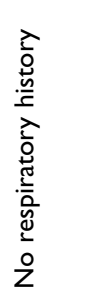 & 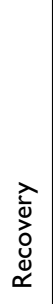 \\
\hline$\stackrel{\sim}{\boldsymbol{n}}$ & 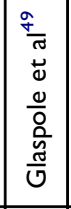 & $\stackrel{m}{\grave{d}}$ & $\stackrel{\circ}{\circ}$ & 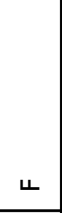 & $\begin{array}{c}\bar{o} \\
z\end{array}$ & 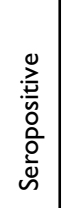 & $\stackrel{\sim}{m}$ & $\mathbb{z}$ & 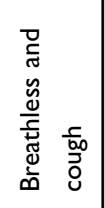 & $\begin{array}{l}\stackrel{\underline{y}}{3} \\
\underline{\simeq}\end{array}$ & $\stackrel{\check{\Xi}}{\longleftarrow}$ & ù & $\mathbb{Z}$ & $\begin{array}{l}\text { 点 } \\
\text { Dू }\end{array}$ \\
\hline$\stackrel{\Delta}{ }$ & 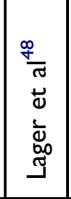 & $\stackrel{m}{\grave{d}}$ & $\approx$ & 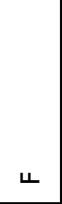 & 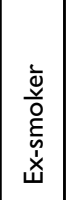 & 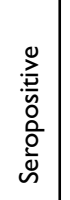 & q & $\mathbb{z}$ & 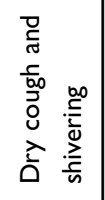 & $\begin{array}{l}\frac{5}{3} \\
\infty\end{array}$ & $\stackrel{\Xi}{\longleftarrow}$ & Ù & $\mathbb{Z}$ & 曾 \\
\hline$\tilde{\boldsymbol{\tau}}$ & 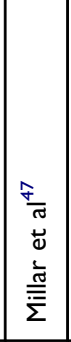 & $\stackrel{\sim}{\circ}$ & $\hat{\omega}$ & $\Sigma$ & $\mathbb{z}$ & 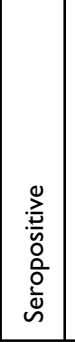 & $\stackrel{+}{m}$ & 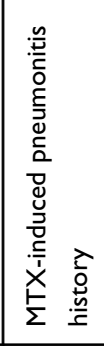 & 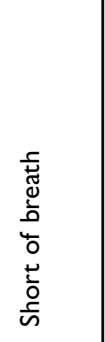 & 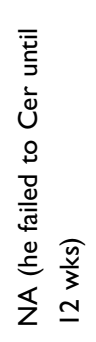 & $\stackrel{\Xi}{\check{\Xi}}$ & Ù & 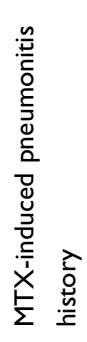 & $\begin{array}{l}\text { 惡 } \\
\text { ه̆ }\end{array}$ \\
\hline $\boldsymbol{N}$ & 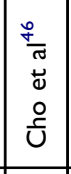 & $\overline{\mathrm{N}}$ & $\stackrel{\circ}{\circ}$ & 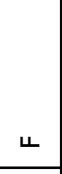 & \begin{tabular}{|l|} 
\\
$z$ \\
\end{tabular} & $\approx$ & $\simeq$ & $\mathbb{z}$ & 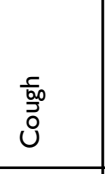 & $\underline{z}$ & $\stackrel{\stackrel{0}{*}}{*}$ & 昰 & $\underline{z}$ & 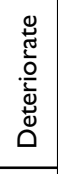 \\
\hline & 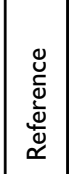 & 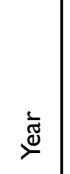 & $\underset{\&}{\mathbb{8}}$ & 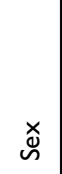 & 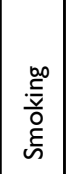 & 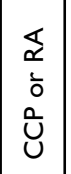 & 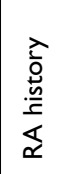 & 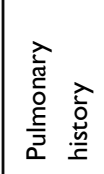 & 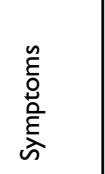 & 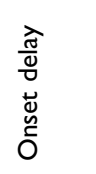 & $\stackrel{x}{\Sigma}$ & 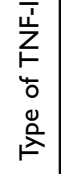 & 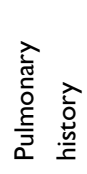 & 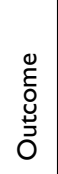 \\
\hline
\end{tabular}


Table 3 Noteworthy differences in case reports

\begin{tabular}{|l|l|l|l|}
\hline & ILD improved (N=5) & ILD induced or deteriorate (N=35) & p-Value \\
\hline Age & $67.4 \pm 3.56$ years & $65.23 \pm 10.16$ years & 0.642 \\
Gender (female) (number/ratio) & $I(25.71 \%)$ & $26(74.29)$ & 0.031 \\
History of RA & $5 \pm 3.56$ years & $15 \pm 4.4$ years & 0.000 \\
Used of MTX (number/ratio) & $3(60 \%)$ & $24(68.37 \%)$ & 1.00 \\
Onset of disease & 1 year & INF $2-3$ wks after $2-3$ doses & - \\
Using INF (number/ratio) & $5(100 \%)$ & $17(48.57 \%)$ & 0.053 \\
CCP or RF positive (number/ratio) & $4(80 \%)$ & $24(68.57 \%)$ & - \\
\hline
\end{tabular}

Abbreviation: ILD, interstitial lung disease; RA, rheumatoid arthritis; MTX, methotrexate; INF, infliximab; CCP cyclic citrullinated peptides; RF, rheumatoid factor.

included more men. Although all ILD benefits were derived from infliximab antibodies, the performance of infliximab in ILD adverse events showed no significant differences compared with other TNF-I drugs.Therefore, more attention should be paid to pulmonary symptoms when TNF-I is prescribed for long-term use in female patients with RA.

\section{Possible decisive factors for different outcomes}

The above results showed that most patients experienced ILD adverse events; however, the outcome was variable. We performed a sub-group analysis (death and survival) of ILD adverse events to explore the decisive factors. The results in Table 4 indicate that deaths accounted for $40 \%$ of the ILD adverse event cases. After the analysis of these results with the chi-square test, significant differences were observed in age, pre-existing lung disease, use of antibiotics, and combination treatment with azathioprine $(p<0.05)$. Patients who died were older (66 vs 64 years) and had a higher use of antibiotics (77\% vs $29 \%$ ). In addition, the combination of azathioprine was positively associated with death. Although the use of MTX might be a risk factor of ILD adverse events, our results showed that the combination of MTX with infliximab probably did not affect ILD. In addition to the quantifiable results, we speculated that patients who died had a more urgent and severe condition based on the median episode delay. Therefore, early detection and precise treatment may be key factors in determining the chances of survival when an ILD adverse event occurs.

\section{Predictors of the probability of onset}

The aforementioned data indicated that timely diagnosis and treatment of ILD might affect outcomes. Therefore, we attempted to identify those predictive features to allow early detection. As shown in Figure 2, infliximab dominance was obvious in both the survival and death groups, whereas

Table 4 Decisive factors of patients receiving TNF-I therapy for different outcomes

\begin{tabular}{|c|c|c|c|c|}
\hline & Death $(N=14)$ & Survivors $(\mathbf{N}=\mathbf{2} \mathrm{I})$ & $p$-Value \\
\hline \multicolumn{2}{|l|}{ Age (years) } & $66.93 \pm 5.59$ & $64.10 \pm 11.96$ & 0.416 \\
\hline \multicolumn{2}{|c|}{ Gender (female) } & 10 (71\%) & $16(76 \%)$ & 1.000 \\
\hline \multicolumn{2}{|l|}{ Smoker } & $2 / 5(40 \%)$ & $6 / 10(60 \%)$ & 0.608 \\
\hline \multirow[t]{2}{*}{ Combination } & INF + MTX & $7(50 \%)$ & $\mid 7(8 \mid \%)$ & 0.073 \\
\hline & $\mathrm{INF}+\mathrm{AZA}$ & $6(43 \%)$ & $\mathrm{I}(5 \%)$ & 0.010 \\
\hline \multicolumn{2}{|l|}{ History of RA } & $15.15 \pm 12.83$ & $14.13 \pm 10.30$ & 0.796 \\
\hline \multicolumn{2}{|c|}{ Median onset delay } & 3 wks & 8 wks & - \\
\hline \multicolumn{2}{|c|}{ Pre-existing lung disease } & $1 \mathrm{I} / 13(79 \%)$ & $6 / 14(47 \%)$ & 0.046 \\
\hline \multicolumn{2}{|c|}{ Joint symptom Remission } & $6 / 8(75 \%)$ & $13 / 14$ (93\%) & 0.527 \\
\hline \multicolumn{2}{|c|}{ Causes of death } & Pulmonary deteriorate: 8 MSOF:I & - & - \\
\hline \multicolumn{2}{|c|}{ Use of antibiotics } & $10(71 \%)$ & $6(29 \%)$ & 0.018 \\
\hline \multicolumn{2}{|c|}{ Without use of prednisolone } & 2 & NA & - \\
\hline
\end{tabular}

Abbreviations: INF, infliximab; AZA, azathioprine; MTX, methotrexate; RA, rheumatoid arthritis; NA, unknown; MSOF, multiple systems organ failure; TNF-I, tumor necrosis factor inhibitor. 
A

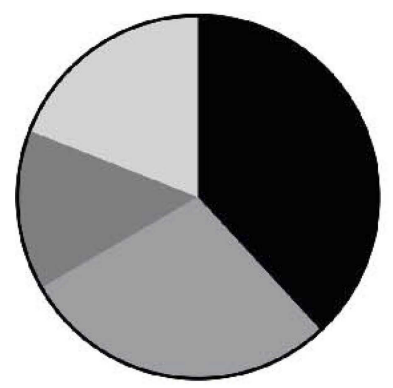

Survivor $(\mathbf{N}=\mathbf{2 1})$
B

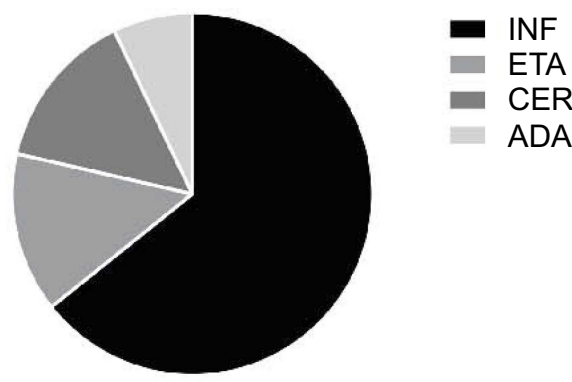

Death $(\mathrm{N}=14)$

Onset of ILD

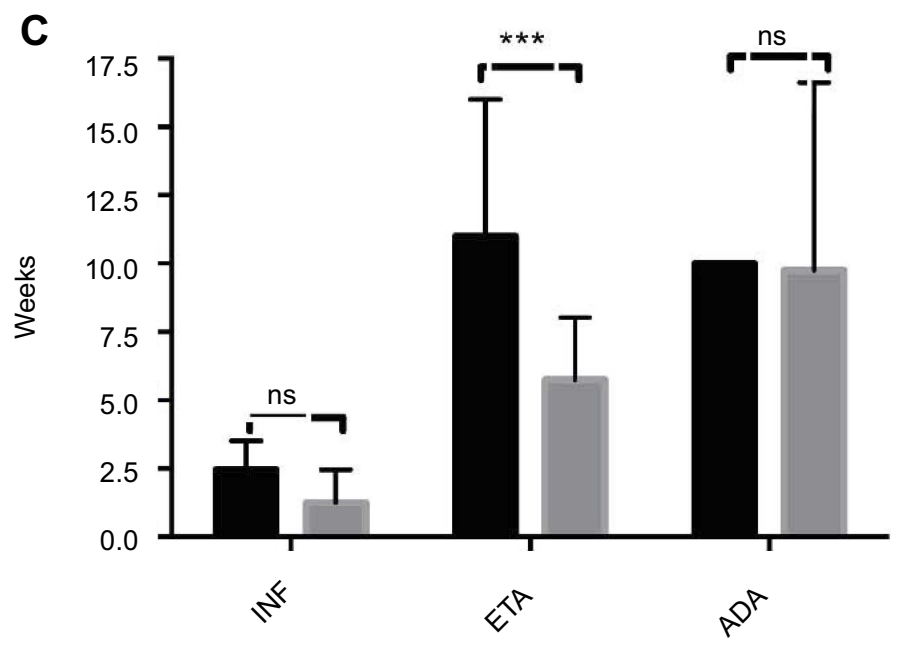

Death

Survivor

Figure 2 Noteworthy differences in case reports. (A) Percentage of different types of biological agents in survivor group; (B) Percentage of different types of biological agents in death group; (C) Comparison of onset time of different types of biological. ${ }^{* * *} p<0.05$.

Abbreviations: ADA, adalimumab; Cer, certolizumab pegol; INF, infliximab; ILD, interstitial lung disease; ETA, etanercept; ns, not significant.

the proportion of adalimumab use was low in the death group. Table 2 demonstrates that the time to death ranged from a few days to 36 weeks; however, Figure $2 \mathrm{C}$ shows significant differences between the survivor and death groups in patients treated with etanercept $(p<0.05)$. Like acute ILD, the most common clinical manifestations in ILD adverse events cases were shortness of breath and sudden dyspnoea $(n=31)$, followed by non-productive cough $(n=17)$. Further, approximately 10 patients were hospitalized with fever. As HRCT is the cornerstone of ILD diagnosis, we searched for related imaging features. The most common patterns were ground glass and reticulation, which were present in more than half of the patients and tended to be sub-pleural and basilar in distribution (Table 2). Surprisingly, the presence of a distinct honeycomb pattern seems to be relatively rare in ILD adverse events. Therefore, we conclude that patients with typical or atypical disease presenting with the groundglass pattern after a few weeks of the last infusion are highly suspicious of having ILD.

\section{Management of ILD adverse events}

Table 4 indicates that inappropriate use of antibiotics might be associated with death. Thus, there is a need to improve the therapy. Some patients recovered after the timely withdrawal of TNF-I and supplementation with high-dose corticosteroids in the acute period, with tapering to lower doses as necessary depending on the condition. However, the empirical use of broad-spectrum antibiotics and increased dose of antibiotics achieved limited effects. In addition, some patients benefited from home oxygen therapy.

\section{Discussion}

Although a better understanding of the pathogenesis of RA and the development of therapies had improved the clinical outcomes in the past decades, the incidence of extraarticular manifestations in patients with RA showed no decrease. ${ }^{2}$ A study also indicated that pulmonary involvement occurs in $60-80 \%$ patients, ${ }^{16}$ and ILD particularly 
greatly affects the morbidity and mortality of RA. ${ }^{3}$ Substantial health-care use and costs were needed with the increasing prevalence of RA- ILD. ${ }^{3}$ Since druginduced ILD is better than idiopathic ILD in prognosis, ${ }^{17}$ there is an urgent need to better identify and avoid the risk factors. As TNF-I is widely used in patients with RA, the safety of TNF-I for ILD needs to be established. In addition to the aforementioned problems, TNF-I-induced ILD not only increases the comorbidities and the mortality rate, but also adds to the difficulty of diagnosis, which, in turn, limits the use of therapeutic drugs. Therefore, we aimed to raise awareness and improve the therapy by evaluating the correlation between TNF-I and ILD in RA.

Previous studies about the relationship between TNF-I and ILD showed controversial results. As TNF- $\alpha$ is known to play a key role in the pathogenesis of ILD, ${ }^{18}$ earlier case reports considered that TNF-I had a positive effect on ILD. In 2004, Bargagli et al $^{16}$ observed the improvement of pulmonary function with 15 months of infliximab treatment, and another study published in 2007 suggested that infliximab might stabilize the progression of ILD in RA. ${ }^{20}$ Clinical results indicated that TNF-I has not increased the incidence of ILD in patients with RA compared with nonbiologic therapies ${ }^{9}$ or alternative mechanism-of-action agents. $^{12}$ However, ever-increasing cases have been reported after the case of fatal exacerbation of RA-ILD in a patient treated with infliximab in $2004 .^{21}$ An analysis ${ }^{22}$ of 122 cases caught our attention owing to the high incidence of ILD after TNF-I (29\%), which is 50 times of that reported in a study using post-marketing surveillance data from Japan $(0.6 \%){ }^{23}$ A later study demonstrated that the proportion of deaths attributable to RA-ILD was higher in patients treated with TNF-I than in those treated with rituximab, ${ }^{14}$ consistent with a cohort study on rituximab. ${ }^{24}$

On the basis of the results, we read the articles and case reports in detail, and found that the controversial results were due to the differences in the population selection, control group, observation period, diagnosis of ILD, and outcome indicators. The current incidence is probably underestimated owing to the rarity of the disease, the short observation period of randomized controlled trials, and the diagnostic methods used to confirm TNF-I associated ILD. The criteria for diagnosing ILD adverse events were as follows: ILD adverse events occurring with an acute or sub-acute lung manifestation shortly after TNF-I infusion with or without remission of articular manifestations, and other related diseases can be excluded on the basis of consistent radiological findings. Notably, patients always do not respond to empiric antibiotic therapy, but symptoms improve when TNF-I is removed. Although the usual interstitial pneumonia pattern accounted for the majority of RA-ILD cases, both pathologically and radiologically, ${ }^{25}$ the non-specific interstitial pneumonia pattern was the most common imaging type in TNFI-associated ILD adverse events. These imaging features indicate an acute onset of ILD that enhances the confidence in the diagnosis. Although azathioprine is the recommended drug for the treatment of RA-ILD, a study ${ }^{26}$ highlighted the risk of infliximab in combination with azathioprine.

Additionally, the fact that nearly $40 \%$ of patients died indicates that detection and precise therapy should not be delayed. Considering the risks and unforeseen results, we believe that rituximab and abatacept might be better choices than TNF-I agents in patients with pre-existing ILD. Once TNF-I is prescribed to patients with RA, more caution should be taken, especially in older patients with a longer RA history or a rheumatoid-associated pulmonary disease. Respiratory symptoms should be evaluated at follow-up. Pulmonary auscultation is recommended as a routine test. Moreover, high-resolution computed tomography of the lung should be conducted as necessary in the first few infusions of TNF-I. When a patient with RA complained of dyspnoea and dry cough without infectious evidence, withdrawing TNF-I and adding prednisolone are recommended. In the presence of fever, night sweats, and other atypical manifestations, ILD should be distinguished from infectious disease as soon as possible. Hence, more mechanisms should be explored and more randomized controlled studies are needed.

Despite the elucidation of the clinical characteristics of ILD adverse events, the pathogenesis remains unclear. Initially, researchers were reluctant to attribute the lung toxicity to TNF-I itself when TNF-I (mainly infliximab) was as a step-up therapy or used in combination with MTX or azathioprine. Perez-Alvarez et $\mathrm{al}^{22}$ postulated that TNF-I potentiates the lung toxicity of MTX, which was then confirmed by an MTX study ${ }^{27}$ and an azathioprine study. ${ }^{28}$ With the ever-increasing use of TNF-I, an increasing incidence of pneumonia toxicity arises. Researchers who considered the status of RA suspected that TNF-I broke the balance (Th1 and Th2, TNF, interferon- $\gamma$, and IL-1) and attributed the adverse effects to inflammatory-mediated pathways. ${ }^{29}$ As another interesting interpretation, Migita et $\mathrm{al}^{30}$ speculated that acute exacerbation of RA-ILD treated with certolizumab 
was due to NLRP3 inflammation activation. Although we found that the onset of ILD adverse events occurred soon after the last infusion, we supposed that the heterogeneous (mouse) of TNF-I might cause allergic reactions and induce ILD indirectly. The separate analysis of articular and lung manifestations suggested that fast remission might make patients poorly tolerant, with pneumonia as one of the manifestations.

Even with the available evidence, it remains difficult to confirm whether TNF-I is beneficial or harmful to ILD owing to the rarity of the disease. However, the everincreasing case reports serve as a reminder that TNF-I might induce more severe symptoms, even death. Evidence showing that some patients with RA developed ILD adverse events indicates that doctors should pay more attention during the first few weeks when introducing TNF-I, especially in old female RA patients with preexisting ILD. Once ILD adverse events occur, precise and timely therapy could be life saving.

\section{Limitations}

We acknowledge that the reported risks of TNF-I-induced ILD varied depending on the study population, control group, drug combinations, outcome indicators, and research duration. Moreover, the heterogeneity of ILD (the slice differences between spontaneous and druginduced ILD) makes it a complex task to interpret the association or causality between TNF-I and ILD. Inevitably, our study has several limitations. First, we attempted to incorporate cohort studies and case reports to increase the power; however, publication bias from the collected articles cannot be excluded. Second, as ILD is a progressive disease, with the first diagnosis occurring after the true incidence, the results of ILD progression might coincide with the TNF-I infusion. Third, because we are overwhelmed with the concern about the occurrence of ILD, the benefit of TNF-I in delaying ILD might have been neglected. Lastly, the assessment of causality is not completely accurate without the mechanisms.

\section{Conclusion}

In summary, patients with RA might not benefit from TNF-I in terms of ILD, and more attention should be paid on TNF-I-associated ILD. Early detection and precise treatment are key factors in determining the chances of survival when an ILD adverse event occurs. A prospective study is needed to establish the association or causality between TNF-I and ILD adverse events. The more mechanisms are known, the more therapies will be developed and the more benefits will be achieved.

\section{Acknowledgments}

This work was supported by the National Natural Science Foundation of China (grant no. 81573802).

\section{Disclosure}

The authors report no conflicts of interest in this work.

\section{References}

1. Castelino FV, Varga J. Interstitial lung disease in connective tissue diseases: evolving concepts of pathogenesis and management. Arthritis Res Ther. 2010;12(4):213. doi:10.1186/ar3097

2. Turesson C, O'Fallon WM, Crowson CS, et al. Extra-articular disease manifestations in rheumatoid arthritis: incidence trends and risk factors over 46 years. Ann Rheum Dis. 2003;62(8):722-727. doi:10.1136/ard.62.8.722

3. Raimundo K, Solomon JJ, Olson AL, et al. Rheumatoid arthritis-interstitial lung disease in the United States: prevalence, incidence, and healthcare costs and mortality. J Rheumatol. 2019;46 (4):360-369.

4. Wells AU, Denton CP. Interstitial lung disease in connective tissue disease-mechanisms and management. Nat Rev Rheumatol. 2014;10 (12):728-739. doi:10.1038/nrrheum.2014.149

5. Roubille C, Haraoui B. Interstitial lung diseases induced or exacerbated by DMARDS and biologic agents in rheumatoid arthritis: a systematic literature review. Semin Arthritis Rheum. 2014;43 (5):613-626. doi:10.1016/j.semarthrit.2013.09.005

6. Dawson JK, Graham DR, Desmond J, et al. Investigation of the chronic pulmonary effects of low-dose oral methotrexate in patients with rheumatoid arthritis: a prospective study incorporating HRCT scanning and pulmonary function tests. Rheumatology (Oxford). 2002;41(3):262-267. doi:10.1093/rheumatology/41.3.262

7. Antoniou KM, Alexandrakis MG, Siafakas NM, et al. Cytokine network in the pathogenesis of idiopathic pulmonary fibrosis. Sarcoidosis Vasc Diffuse Lung Dis. 2005;22(2):91-104.

8. Kahler CM, Heininger P, Loeffler-Ragg J, et al. Infliximab therapy in pulmonary sarcoidosis. Am J Respir Crit Care Med. 2007;176 (4):417; author reply 417-418.

9. Herrinton LJ, Harrold LR, Liu L, et al. Association between anti-TNFalpha therapy and interstitial lung disease. Pharmacoepidemiol Drug Saf. 2013;22(4):394-402. doi:10.1002/pds.3409

10. Nakashita T, Ando K, Kaneko N, Takahashi K, Motojima S. Potential risk of TNF inhibitors on the progression of interstitial lung disease in patients with rheumatoid arthritis. BMJ Open. 2014;4(8):e005615. doi:10.1136/bmjopen-2014-005615

11. Nakashita T, Ando K, Takahashi K, Motojima S. Possible effect of abatacept on the progression of interstitial lung disease in rheumatoid arthritis patients. Respir Investig. 2016;54(5):376-379. doi:10.1016/j. resinv.2016.03.001

12. Curtis JR, Sarsour K, Napalkov P, Costa LA, Schulman KL. Incidence and complications of interstitial lung disease in users of tocilizumab, rituximab, abatacept and anti-tumor necrosis factor alpha agents, a retrospective cohort study. Arthritis Res Ther. 2015;17:319. doi:10.1186/s13075-015-0835-7

13. Koo BS, Hong S, Kim YJ, et al. Mortality in patients with rheumatoid arthritis-associated interstitial lung disease treated with an anti-tumor necrosis factor agent. Korean J Intern Med. 2015;30 (1):104-109. doi:10.3904/kjim.2015.30.1.104 
14. Druce KL, Iqbal K, Watson KD, et al. Mortality in patients with interstitial lung disease treated with rituximab or TNFi as a first biologic. RMD Open. 2017;3(1):e000473. doi:10.1136/rmdopen2016-000391

15. Detorakis EE, Magkanas E, Lasithiotaki I, et al. Evolution of imaging findings, laboratory and functional parameters in rheumatoid arthritis patients after one year of treatment with anti-TNF-alpha agents. Clin Exp Rheumatol. 2017;35(1):43-52.

16. Cortet B, Perez T, Roux N, et al. Pulmonary function tests and high resolution computed tomography of the lungs in patients with rheumatoid arthritis. Ann Rheum Dis. 1997;56(10):596-600. doi:10.1136/ ard.56.10.596

17. Johnson C. Recent advances in the pathogenesis, prediction, and management of rheumatoid arthritis-associated interstitial lung disease. Curr Opin Rheumatol. 2017;29(3):254-259. doi:10.1097/ BOR.0000000000000380

18. Panopoulos ST, Sfikakis PP. Biological treatments and connective tissue disease associated interstitial lung disease. Curr Opin Pulm Med. 2011;17(5):362-367. doi:10.1097/MCP.0b013e3283483ea5

19. Bargagli E, Galeazzi M, Rottoli P. Infliximab treatment in a patient with rheumatoid arthritis and pulmonary fibrosis. Eur Respir J. 2004;24(4):708. doi:10.1183/09031936.04.00076904

20. Antoniou KM, Mamoulaki M, Malagari K, et al. Infliximab therapy in pulmonary fibrosis associated with collagen vascular disease. Clin Exp Rheumatol. 2007;25(1):23-28.

21. Ostor AJ, Crisp AJ, Somerville MF, Scott DGI. Fatal exacerbation of rheumatoid arthritis associated fibrosing alveolitis in patients given infliximab. BMJ (Clinical Research Ed). 2004;329(7477):1266. doi:10.1136/bmj.329.7477.1266

22. Perez-Alvarez R, Perez-de-Lis M, Diaz-Lagares C, et al. Interstitial lung disease induced or exacerbated by TNF-targeted therapies: analysis of 122 cases. Semin Arthritis Rheum. 2011;41(2):256-264. doi:10.1016/j.semarthrit.2010.11.002

23. Koike T, Harigai M, Ishiguro N, et al. Safety and effectiveness of adalimumab in Japanese rheumatoid arthritis patients: postmarketing surveillance report of the first 3,000 patients. Mod Rheumatol. 2012;22(4):498-508. doi:10.3109/s10165-011-0541-5

24. Md Yusof MY, Kabia A, Darby M, et al. Effect of rituximab on the progression of rheumatoid arthritis-related interstitial lung disease: 10 years' experience at a single center. Rheumatology (Oxford). 2017;56 (8):1348-1357.

25. Kim EJ, Collard HR, King TE Jr. Rheumatoid arthritis-associated interstitial lung disease: the relevance of histopathologic and radiographic pattern. Chest. 2009;136(5):1397-1405. doi:10.1378/ chest.09-0444

26. Ostor AJ, Chilvers ER, Somerville MF, et al. Pulmonary complications of infliximab therapy in patients with rheumatoid arthritis. $J$ Rheumatol. 2006;33(3):622-628.

27. Villeneuve E, St-Pierre A, Haraoui B. Interstitial pneumonitis associated with infliximab therapy. J Rheumatol. 2006;33(6):1189-1193.

28. Bedrossian CW, Sussman J, Conklin RH, et al. Azathioprineassociated interstitial pneumonitis. Am J Clin Pathol. 1984;82 (2):148-154. doi:10.1093/ajcp/82.2.148

29. Alaee S, Jones Q. Case of drug-induced interstitial lung disease secondary to adalimumab. BMJ Case Rep. 2018;pii:bcr-2018224375.

30. Migita K, Tsuji Y, Hisatomi K, et al. Acute exacerbation of rheumatoid interstitial lung disease during the maintenance therapy with certolizumab pegol. Modern Rheumatology. 2017;27(6):1079-1082. doi:10.3109/14397595.2015.1059008

31. Peno-Green L, Lluberas G, Kingsley T, Brantley S. Lung injury linked to etanercept therapy. Chest. 2002;122(5):1858-1860.
32. Vassallo R, Matteson E, Thomas CF, Jr. Clinical Response of Rheumatoid Arthritis-Associated Pulmonary Fibrosis to Tumor Necrosis factor-alpha Inhibition. Chest. 2002;122(3):1093-1096.

33. Chatterjee, S. Severe interstitial pneumonitis associated with infliximab therapy. Scand J Rheumatol. 2004;33(4):276-277.

34. Hennum J, Nace J, Shammash E, Gertner E. Infliximab-associated pneumonitis in rheumatoid arthritis. J Rheumatol. 2006;33(9):19171918.

35. Mori S, Imamura F, Kiyofuji C, Sugimoto M. Development of interstitial pneumonia in a rheumatoid arthritis patient treated with infliximab, an anti-tumor necrosis factor alpha-neutralizing antibody. Mod Rheumatol. 2006;16(4):251-255.

36. Lindsay K, Melsom R, Jacob BK, Mestry N. Acute progression of interstitial lung disease: a complication of etanercept particularly in the presence of rheumatoid lung and methotrexate treatment. Rheumatology (Oxford). 2006;45(8):1048-1049.

37. Schoe A, van der Laan-Baalbergen NE, Huizinga TW, Breedveld FC, van Laar JM. Pulmonary fibrosis in a patient with rheumatoid arthritis treated with adalimumab. Arthritis Rheum. 2006;55(1):157-159.

38. Huggett MT, Armstrong R. Adalimumab-associated pulmonary fibrosis. Rheumatology (Oxford). 2006;45(10):1312-1313.

39. Courtney PA, Alderdice J, Whitehead EM. Comment on methotrexate pneumonitis after initiation of infliximab therapy for rheumatoid arthritis. Arthritis Rheum. 2003;49(4):617; 617-618.

40. Hagiwara K, Sato T, Takagi-Kobayashi S, Hasegawa S, Shigihara N, Akiyama O. Acute exacerbation of preexisting interstitial lung disease after administration of etanercept for rheumatoid arthritis. $J$ Rheumatol. 2007;34(5):1151-1154.

41. Tournadre A, Ledoux-Eberst J, Poujol D, Dubost JJ, Ristori JM, Soubrier M. Exacerbation of interstitial lung disease during etanercept therapy: Two cases. Joint Bone Spine. 2008;75(2):215-218.

42. Taki H, Kawagishi Y, Shinoda K, et al. Interstitial pneumonitis associated with infliximab therapy without methotrexate treatment. Rheumatol Int. 2009;30(2):275-276.

43. Pearce F, Johnson SR, Courtney P. Interstitial lung disease following certolizumab pegol. Rheumatology (Oxford). 2012;51(3):578-580.

44. Yamazaki H, Isogai S, Sakurai T, Nagasaka K. A case of adalimumab-associated interstitial pneumonia with rheumatoid arthritis. Mod Rheumatol. 2010;20(5):518-521.

45. Komiya K, Ishii H, Fujita N, et al. Adalimumab-induced interstitial pneumonia with an improvement of pre-existing rheumatoid arthritisassociated lung involvement. Intern Med. 2011;50(7):749-751.

46. Cho SK, Oh IH, Park CK, Bae SC, Sung YK. Etanercept induced organizing pneumonia in a patient with rheumatoid arthritis. Rheumatol Int. 2012;32(4):1055-1057.

47. Millar A, McKew J, Taggart A. Fatal fibrosing alveolitis with certolizumab. Rheumatology (Oxford). 2012;51(5):953-955.

48. Lager J, Hilberg O, Lokke A, Bendstrup E. Severe interstitial lung disease following treatment with certolizumab pegol: a case report. European respiratory review: an official journal of the European Respiratory Society. 2013;22(129):414-416.

49. Glaspole IN, Hoy RF, Ryan PF. A case of certolizumab-induced interstitial lung disease in a patient with rheumatoid arthritis. Rheumatology (Oxford). 2013;52(12):2302-2304.

50. Dias OM, Pereira DA, Baldi BG, et al. Adalimumab-induced acute interstitial lung disease in a patient with rheumatoid arthritis. Jornal brasileiro de pneumologia : publicacao oficial da Sociedade Brasileira de Pneumologia e Tisilogia. 2014;40(1):77-81.

51. Watad A, Perelman M, Mansour R, Shoenfeld Y, Amital H. Etanercept-Induced Pneumonitis: Severe Complication of Tumor Necrosis Factor-Alpha Blocker Treatment. The Israel Medical Association journal: IMAJ. 2015;17(2):130-132. 


\section{Publish your work in this journal}

Drug Design, Development and Therapy is an international, peerreviewed open-access journal that spans the spectrum of drug design and development through to clinical applications. Clinical outcomes, patient safety, and programs for the development and effective, safe, and sustained use of medicines are a feature of the journal, which has also

been accepted for indexing on PubMed Central. The manuscript management system is completely online and includes a very quick and fair peer-review system, which is all easy to use. Visit http://www. dovepress.com/testimonials.php to read real quotes from published authors. 